Jahrbuch Schweiz - Dritte Welt 1996

\title{
Die Ernährung der Menschheit im 21. Jahrhundert
}

Die Politik der FAO zur Bekämpfung des Hungers

Haltung der Schweiz

Anton Kohler

\section{(2) OpenEdition}

12 Journals

Electronic version

URL: http://journals.openedition.org/sjep/1357

DOI: 10.4000/sjep.1357

ISSN: 1663-9677

Publisher

Institut de hautes études internationales et du développement

\section{Printed version}

Date of publication: 1 mars 1996

Number of pages: 273-289

ISSN: 1660-5926

\section{Electronic reference}

Anton Kohler, « Die Ernährung der Menschheit im 21. Jahrhundert », Schweizerisches Jahrbuch für Entwicklungspolitik [Online], 15 | 1996, Online erschienen am: 18 Mai 2013, abgerufen am 10 Dezember 2020. URL : http://journals.openedition.org/sjep/1357 ; DOI : https://doi.org/10.4000/sjep.1357 


\title{
Die Ernährung der Menschheit im 21. Jahrhundert
}

\section{Die Politik der FAO zur Bekämpfung des Hungers}

\section{Haltung der Schweiz}

\author{
Anton Kohler
}

\section{Gegenwart und Zukunft}

Am Ende des 20. Jahrhunderts bleibt eine der zentralsten Fragen der Menschheit unbeantwortet: Wird es gelingen, für eine wachsende Weltbevölkerung genügend Nahrungsmittel zu produzieren und damit den Hunger zu eliminieren?

Die Meinungen der Wissenschafter zu dieser Frage gehen stark auseinander. Pessimisten mit oft malthusianischen Perspektiven stehen der Gruppe der Optimisten gegenüber, die insbesondere von der Bio- und Gentechnologie wesentliche Fortschritte erwarten, während erstere vor allem aus ökologischen Bedenken Zweifel an der Nachhaltigkeit landwirtschaftlicher Expansion äussern. Der Generaldirektor der FAO, Jacques Diouf, baut auf künftige Errungenschaften einer "Nachhaltigen Neuen Grünen Revolution“. Im folgenden soll die Strategie der FAO zur Verbesserung der Ernährungssicherheit vor allem in den ärmsten Ländern der Welt dargestellt werden.

\subsection{Die Ausgangslage im Jahre 1995}

Um die Welternährungslage steht es heute besser bestellt als noch vor 25 Jahren. Im Jahre 1970 litten ca. 950 Millionen Menschen an Hunger und Unterernährung. Heute wird deren Zahl auf 800 Millionen geschätzt. Im selben Zeitraum ist die Weltbevölkerung um 2,5 Milliarden auf heute ca. 5,7 Milliarden Menschen angewachsen. Die Verbesserung der Ernährungs- 
situation stellt einen beträchtlichen Erfolg von Eigen-anstrengungen in Entwicklungsländern gepaart mit den Leistungen der Entwicklungszusammenarbeit dar! Doch an der Schwelle zum 21. Jahrhundert steht die Menschheit vor dem Dilemma einer immer noch schnell wachsenden Weltbevölkerung mit prekärer werdender Ausstattung an natürlichen Ressourcen. Wie soll also das Recht auf genügende Ernährung für alle Menschen Realität werden? Der Generaldirektor der FAO, Jacques Diouf, hat bereits nach seiner Wahl im November 1993 das Problem weltweiten Hungers als inakzeptable menschliche Tragödie bezeichnet. Nach wie vor leiden mehr als 200 Millionen Kinder unter 5 Jahren an chronischem Hunger und akutem Proteinmangel. Noch heute sterben jährlich 15 Mio Kinder an Hunger.

\section{2. "Horizon $2010 " 1$}

Mitte 1995 publizierte die FAO unter dem Titel „Horizon 2010“ eine breitangelegte Analyse zur Zukunft der Ernährung der Menschheit. Das beschränkte Potential der Erde und die künftigen Möglichkeiten der Landwirtschaft, eine stetig wachsende Menschheit bis zum Jahr 2010 mit genügend Nahrungsmitteln zu versorgen, stehen im Zentrum der Analyse. Diese kommt zu folgenden Schlüssen:

- Die Erdbevölkerung wird bis zum Jahre 2010 von gegenwärtig 5.7 Milliarden auf ca. 7 Milliarden Menschen ansteigen. Die durchschnittliche Wachstumsrate, welche von Region zu Region grosse Unterschiede aufweist, beträgt $1,6 \%$ pro Jahr. Mit Ausnahme von Afrika wird die Zuwachsrate kontinuierlich sinken.

- Das Wachstum der Agrarproduktion wird sich weiter verlangsamen. Statt wie in den vergangenen 20 Jahren um 2,3\%, wird die Nahrungsmittelerzeugung bis zum Jahr 2010 jährlich lediglich noch um 1,8\% zunehmen. Sie wird damit nur wenig höher als das Bevölkerungswachstum ausfallen. Die jährliche Zuwachsrate der Agrarproduktion pro Einwohner im Zeitraum 1970-1990 von 0,54\% wird sich in den Jahren 1990-2010 auf $0,25 \%$ verringern.

- In den Entwicklungsländern leben heute über eine Milliarde Menschen unter der Armutsgrenze mit einem Tageseinkommen pro Kopf von weniger als 1 Dollar. In Südasien und in Afrika betrifft dies etwa $50 \%$ der Bevölkerung. 2 Milliarden Menschen müssen mit einem Tageseinkommen von 2 Dollars oder weniger auskommen.

- Wegen der andauernden Armut wird die marktwirksame Nachfrage nach Nahrungsmitteln langsamer wachsen als bisher, auch wenn der physische Bedarf markant steigen wird. Doch auch das geringere Bevölkerungswachstum und die Saturierung im reicheren Teil der Welt trägt zur Abschwächung der Nachfrage bei. Starke Produktionsanreize für die Bauern werden daher fehlen.

1. Horizon 2010: Agriculture towards 2010, FAO, Rom 1995 


\begin{tabular}{|c|c|c|c|c|}
\hline \multicolumn{5}{|c|}{$\begin{array}{l}\text { Wachstumsraten von Bevölkerung und Nahrungsmittelproduktion } \\
\text { weltweit (1970-1990 und Prognose für 1990-2010) }\end{array}$} \\
\hline \multicolumn{2}{|l|}{ Länder } & $\begin{array}{l}\text { Bevölke- } \\
\text { rungs- } \\
\text { wachstum }\end{array}$ & $\begin{array}{l}\text { Wachstum } \\
\text { der } \\
\text { Nahrungs- } \\
\text { mittel- } \\
\text { produktion* }\end{array}$ & $\begin{array}{l}\text { Wachstum } \\
\text { der } \\
\text { Produktion } \\
\text { pro Kopf* }^{\star}\end{array}$ \\
\hline & & $\%$ pro Jahr & $\%$ pro Jahr & $\%$ pro Jahr \\
\hline Welt & $\begin{array}{l}1970-1990 \\
1990-2010\end{array}$ & $\begin{array}{l}1,8 \\
1,6\end{array}$ & $\begin{array}{l}2,3 \\
1,8\end{array}$ & $\begin{array}{l}0,54 \\
0,25\end{array}$ \\
\hline Industrieländer & $\begin{array}{l}1970-1990 \\
1990-2010\end{array}$ & $\begin{array}{l}0,8 \\
0,5\end{array}$ & $\begin{array}{l}1,4 \\
0,7\end{array}$ & $\begin{array}{l}0,6 \\
0,2\end{array}$ \\
\hline $\begin{array}{l}\text { Entwicklungsländer } \\
\text { (inkl. China) } \\
\text { davon: }\end{array}$ & $\begin{array}{r}1970-1990 \\
1990-2010\end{array}$ & $\begin{array}{l}2,2 \\
1,8\end{array}$ & $\begin{array}{l}3,3 \\
2,6\end{array}$ & $\begin{array}{l}1,1 \\
0,8\end{array}$ \\
\hline Schwarzafrika & $\begin{array}{l}1970-1990 \\
1990-2010\end{array}$ & $\begin{array}{l}3,0 \\
3,2\end{array}$ & $\begin{array}{l}1,9 \\
3,0\end{array}$ & $\begin{array}{l}-1,1 \\
-0,2\end{array}$ \\
\hline $\begin{array}{l}\text { Naher Osten } \\
+ \text { Nordafrika }\end{array}$ & $\begin{array}{l}1970-1990 \\
1990-2010\end{array}$ & $\begin{array}{l}2,8 \\
2,4\end{array}$ & $\begin{array}{l}3,1 \\
2,7\end{array}$ & $\begin{array}{l}0,3 \\
0,3\end{array}$ \\
\hline Lateinamerika & $\begin{array}{l}1970-1990 \\
1990-2010\end{array}$ & $\begin{array}{l}2,3 \\
1,7\end{array}$ & $\begin{array}{l}2,9 \\
2,3\end{array}$ & $\begin{array}{l}0,6 \\
0,6\end{array}$ \\
\hline Asien Ost & $\begin{array}{l}1970-1990 \\
1990-2010\end{array}$ & $\begin{array}{l}1,7 \\
1,2\end{array}$ & $\begin{array}{l}4,1 \\
2,7\end{array}$ & $\begin{array}{l}2,4 \\
1,5\end{array}$ \\
\hline Asien Süd & $\begin{array}{l}1970-1990 \\
1990-2010\end{array}$ & $\begin{array}{l}2,4 \\
2,0\end{array}$ & $\begin{array}{l}3,1 \\
2,6\end{array}$ & $\begin{array}{l}0,7 \\
0,6\end{array}$ \\
\hline
\end{tabular}

- Die Hungerproblematik verlagert sich schwergewichtigtsmässig von Südasien ins Afrika südlich der Sahara, wo die Anzahl hungernder Menschen bis zum Jahr 2010 von heute 175 Millionen auf 300 Millionen ansteigen wird. Dies werden $32 \%$ der Einwohner dieses Kontinents oder $50 \%$ aller Hungernden der Welt sein. In Südasien werden ca. $12 \%$ der Einwohner an Hunger leiden. Die Anzahl Hungernder in Afrika südlich der Sahara wird jene in Südasien übertreffen. Selbst bei zusätzlichen Anstrengungen wird die chronische Unterernährung in beiden Regionen bis zum Jahr 2010 kaum zu überwinden sein. 
- Angesichts des Bevölkerungswachstums wird sich der Konflikt zwischen Entwicklung, Ernährung und Umwelt verschärfen. Die Knappheit von Boden und Wasser wird drastisch zunehmen und der Druck auf die verfügbaren natürlichen Ressourcen sich akzentuieren. Die landwirtschaftliche Flächenausdehnung in den Entwicklungsländern wird bis zum Jahr 2010 an ihre Grenzen stossen.

- Fortbestehen von Armut, ungünstige Ausstattung der Problemländer und regionen mit natürlichen und finanziellen Ressourcen und schlechte Aussichten für deren ökonomische und soziale Entwicklung werden die landwirtschaftliche Produktion weiterhin stark hemmen.

\section{Die Herausforderung}

Das Hauptziel der FAO, die Menschheit vom Hunger zu befreien, wurde anlässlich des 50-jährigen Bestehens der UN-Organisation in der "Erklärung von Québec" vom 16. Oktober 1995 von allen Mitgliedländern bekräftigt. Vor allem in den ärmsten Entwicklungsländern soll sich die Ernährungssicherheit wesentlich verbessern. Dem von den Vereinten Nationen und ihren Mitgliedländern proklamierten Grundrecht zur Freiheit von Hunger soll vermehrt Achtung verschafft werden.

\subsection{FAO-Ziele zur Hungerbekämpfung}

Aus verschiedenen FAO Quellen geht hervor, dass sich die FAO zum Ziel gesetzt hat, folgende Herausforderungen bis zum Jahre 2010 zu bewältigen:

- Die FAO will wesentlich dazu beitragen, auf die Zahl der chronisch Unterernährten von heute rund 800 Millionen Menschen bis zum Jahr 2010 signifikant unter 650 Millionen zu senken.

- Die FAO strebt in den Entwicklungsländern bis zum Jahr 2010 eine Verbesserung der durchschnittlichen Pro-Kopf Versorgung mit Nahrungsenergie von $2500 \mathrm{kcal} / \mathrm{Tag}$ im Jahre 1995 auf $2700 \mathrm{kcal} / \mathrm{Tag} \mathrm{im}$ Jahre 2010 an. (Der Nahe Osten, Ostasien einschliesslich China, Lateinamerika und die Karibik werden in den nächsten 20 Jahren wahrscheinlich die $\mathbf{3 0 0 0}$ Kalorien-Marke erreichen oder überschreiten können.)

- Zur Bekämpfung einer sich verschärfenden Ernährungslage und zur Erhöhung der Ernährungssicherheit in den einkommensschwachen Nahrungsdefizit-Ländern schlägt die FAO eine Sonderstrategie vor.

- Gemäss dieser Strategie müssen zwei Drittel der Mehrproduktion von Nahrung aus nachhaltigen Produktivitätssteigerungen ertragsstarker Regionen mit höheren Erträgen resultieren.

Um diese Ziele zu erreichen, muss die Entwicklung der Landwirtschaft in den ärmsten Ländern wieder einen zentralen Platz einnehmen. Für drei Viertel der Bevölkerung armer Länder ist der primäre Sektor einzige Quelle 
von Produktion und Einkommen. Die Agrarwirtschaft hat eine volkswirtschaftliche Schlüsselfunktion. Die Förderung einer nachhaltigen Landwirtschaft, Fischerei und Waldwirtschaft ist daher unumgänglich zur Einkommenssteigerung, zum Abbau der Armut, zur Reduktion des Hungers, zur Verbesserung der Ernährungssicherheit und für den sozialen Fortschritt. Eine Wende in der globalen Ernährungssituation ist nur vorstellbar, wenn die Leistungsfähigkeit der Landwirtschaft, von der die Volkswirtschaft in hohem Masse abhängt, vor allem in den ärmsten Entwicklungsländern, verbessert wird.

\subsection{Ernährungssicherheit für alle}

Die obgenannten Ziele orientieren sich am strategischen Oberziel der FAO: Die Ernährungssicherheit weltweit, vor allem aber in den ärmsten Ländern zu verbessern. Ernährungssicherheit garantiert allen Menschen das Grundrecht auf die physische Versorgung und den ökonomischen Zugang zu Grundnahrungsmitteln. Zur Verbesserung der Ernährungssicherheit gilt es daher, eine zukunftsweisende, erfolgversprechende Strategie zu entwickeln².

\subsection{Die einkommensschwachen Nahrungsdefizitländer (LIFDC's)}

Um die Ernährungssicherheit ist es in erster Linie in den einkommensschwachen Nahrungs-defizitländern schlecht bestellt. Armut und Hunger erfassen in diesen Ländern weite Bevölkerungskreise. Diese unter dem Begriff der Low Income Food Deficit Countries (LIFDC's) zusammengefassten Länder weisen eine niedrige Selbstversorgungsbasis, kombiniert mit einem sehr tiefen Pro Kopf Einkommen, auf.

- Gemäss FAO zählten im Jahre 199588 Länder mit über 3,5 Milliarden Einwohnern zur Gruppe der LIFDCs. In diesen Ländern ist die Eigenproduktion an Nahrungsmitteln erheblich hinter dem stark gewachsenen Bedarf an Nahrung infolge Bevölkerungszunahme zurückgeblieben. Um die Ernährungssicherheit ist es vor allem in Afrika südlich der Sahara schlecht bestellt. Importmöglichkeiten sind mangels volkswirtschaftlicher Alternativen zur Devisenbeschaffung stark eingeschränkt. Die Abhängigkeit der LIFDC's Afrikas von externer, ungesicherter Nahrungsmittelhilfe hat steigende Tendenz.

2. Die Ernährungssicherheit wird von der FAO wie folgt definiert:

"Que la nourriture soit disponible à tout moment, que tout le monde puisse y avoir accès, qu'elle soit adaptée du point de vue nutritionnel tant en quantité qu'en qualité et en variété, et qu'elle soit acceptable sur le plan culturel“ ("l'autodépendance“, contrairement à "l'autosuffisance", couvre à la fois la production intérieur et la capacité d'importer pour satisfaire les besoins de la population.. L'autodépendance est un objectif non seulement au niveau de la planète et des pays, mais aussi au sein de chaque foyer.) 
- 8 Länder weisen einen extrem tiefen Grad an Ernährungssicherheit auf (Mozambique, zentralafrikan. Republik, Afghanistan, Tschad, Somalia, Burundi, Äthiopien, Eritrea). Aus rein agrartechnischer Perspektive bestünde in diesen Ländern aber laut der Studie "Horizon 2010" ein landwirtschaftliches Produktionspotential, das eine ausreichende Selbstversorgung mit Nahrungsmitteln bis zum Jahr 2010 sichern könnte.

- Von 40 Ländern mit einem tiefen oder sehr tiefen Grad an Ernährungssicherheit sind 21 afrikanische Staaten. Sie weisen aber alle ein gutes landwirtschaftliches Entwicklungspotential auf. Bei voller Nutzung des agrartechnischen Potentials könnte bis zum Jahr 2010 in diesen Ländern mehr als das Doppelte an benötigten Nahrungsmitteln produziert werden.

\section{Potentiale und Grenzen: Entwicklung einer Strategie}

\subsection{Das Dilemma}

Der Bericht "Zur Lage der Welt 1995"3 umschreibt das Dilemma der Menschheit am Übergang vom 20. zum 21. Jahrhundert folgendermassen: "Lautlos und ohne grosses Aufsehen ist die Welt in ein neues Zeitalter eingetreten, und dieses ist geprägt von der Unsicherheit darüber, wie man die voraussichtlich stark wachsende Weltbevölkerung ernähren soll“.

Um Wege aus dieses Situation und Mittel zur Verbesserung der Ernährungssicherheit zu finden, erarbeitete die FAO ein umfassendes Analyseinstrument. Mit dessen Hilfe können agrar-ökologische Potentiale inventarisiert und bestehende Engpässe identifiziert werden.

\subsection{Die Grenzen der Natur}

Die Grenzen des Wachstums, vom Club of Rome im Jahr 1972 vor allem im Energiebereich geortet, nehmen bei Land, Wasser und Wald immer dramatischere Konturen an. Im agro-ökologischen Bereich stellen sich folgende Probleme:

\section{- begrenzte landwirtschaftliche Flächen und deren agro- ökologische Grenzen}

Waren im Jahre 1950 pro Kopf der Bevölkerung noch 0.23 Hektaren Kulturland zur landwirtschaftlichen Nutzung verfügbar, so verblieben im Jahre 1993 nur noch 0.13 Hektaren. Dieser Schrumpfungsprozess geht weiter und spitzt sich zu, weil die Intensivierung der Produktion schnell an natürliche Grenzen stösst und die Bodenproduktivität abnehmende Tendenz aufweist. Zwar lässt sich mittels erhöhtem Düngereinsatz in vielen Entwicklungsländern der Hektarertrag noch steigern, doch ist dies mit beträchtlichen ökologischen

3. Zur Lage der Welt - 1995, S. 12; Fischer Taschenbuch 12895, Frankfurt a.M. 1995 
Risiken verbunden. Jährlich gehen weltweit wegen Übernutzung und Erosion zwischen 5 und 7 Millionen Hektaren gutes Kulturland verloren. Diese Verluste können gemäss FAO-Berechnungen nur knapp wettgemacht werden durch noch verfügbare Landreserven.

Das weltweite FAO-Inventar über sieben agro-ökologische Landtypen erlaubt es, die landwirtschaftlichen Produktionspotentiale und Limiten zu schätzen. (1) In den Entwicklungsländern (ohne China) stehen gesamthaft 2570 Millionen Hektaren Land mit einem sehr guten bis hin zu einem marginalen landwirtschaftlichen Produktionspotential zur Verfügung. Hiervon sind heute ca. 760 Millionen Hektaren bereits landwirtschaftlich genutzt. Von den verbleibenden 1800 Millionen Hektaren erweisen sich lediglich 90 Millionen Hektaren als für die landwirtschaftliche Nutzung geeignet. Die verbleibenden Reserven sind überwiegend Land sehr tiefer Produktivität, Böden in Hanglagen, Wälder und Naturschutzgebiete.

\section{- Wasser}

30 Prozent der Bevölkerung in den Entwicklungsländern verfügen immer noch nicht über gesichertes sauberes Trinkwasser. Aber auch für die Steigerung der landwirtschaftlichen Bodenproduktivität gilt Wasser heute als knappster Faktor. Es kommt hinzu, dass grössere Bewässerungssysteme nicht effizient genug genutzt werden, weil die Bauern in Bewässerungsperimetern für das Wasser oft einen zu tiefen oder gar keinen Preis bezahlen. Die Ungleichheiten zwischen Reich und Arm, zwischen regenabhängiger Trockenlandwirtschaft und bewässerter Landwirtschaft werden dadurch verstärkt, weil Wasser nur selten besteuert wird. In Regionen mit Bewässerung aus Grundwasser sinkt der Pegel wegen Übernutzung durch Motorpumpen.

\section{- Wald}

Zwischen 1980 und 1990 wurden im Durchschnitt jährlich 15,4 Millionen Hektaren tropischer Regenwald zerstört. Der Prozess nicht nachhaltiger Holzgewinnung und anschliessender unsachgemäßer Bodennutzung darf nicht den Marktkräften allein überlassen werden, denn die 30 Prozent noch bewaldeter Flächen sind ernsthaft gefährdet und stehen nicht zuletzt von Seiten der Landwirtschaft unter enormem Druck.

\section{3 Ökonomische Grenzen, soziokulturelle Widerstände und institutionelle Hindernisse}

In ihrer Analyse zum Entwurf neuer Produktionsstrategien geht die FAO auch auf Engpässe und Widrigkeiten ökonomischer, infrastruktureller und institutioneller Art ein. Letztere Kategorie umfasst selbstredend auch den Problemkreis des "Good Governance", wie er etwa aus dem Leitbild Nord-Süd der schweizerischen Entwicklungszusammenarbeit bekannt ist. 
Sozio-kulturelle Widerstände gegenüber landwirtschaftlichen Innovationen und Hindernisse für die Produktivitätssteigerung finden sich auch in der Agrarstruktur (Grundbesitzverhältnisse Pachtsysteme, u.a.), im Arbeitsmarkt und in den Anstellungsverhältnissen von Arbeitskräften, im Ausbildungsniveau und insbesondere in der mangelnden Förderung der Frau im Entwicklungsprozess. All diese Faktoren werden zu Recht als sehr ausschlaggebend für das Ausmass der Akzeptanz gegenüber neuen Technologien und für die Art und Weise der Nutzung des agrar-ökologischen Potentials erachtet. Aus Platzgründen kann auf die Erörterung dieser Thematik nicht näher eingegangen werden. Man kann sich allerdings des Eindruckes nicht erwehren, dass die FAO die Schwierigkeiten im Umgang mit diesen limitierenden Faktoren unterschätzt und insbesondere in Afrika allzu optimistisch auf deren Überwindbarkeit setzt.

\subsection{Wissenschaftliche Neuerungen}

Das Einsatzpotential neuer Bio- und Gentechnologien in der regenabhängigen wie auch in der bewässerten Landwirtschaft stellt eine grosse Unbekannte in der künftig geltenden Produktionsfunktion dar. Die FAO rechnet in nächster Zukunft mit neuen wissenschaftlichen Erkenntnissen und Durchbrüchen, die zu neuen landwirtschaftlichen Innovationen führen dürften, die allerdings das Produktivitätspotential der sog. Grünen Revolution kaum erreichen werden. In inren Strategien setzt die FAO auch gezielt auf in Wissenschaft und Praxis bewährte Methoden zur Verlustminimierung, wie z.B. der erfolgreichen biologischen Bekämpfung von Pflanzen Zenschädlingen (Integrated Pest Management, IPM).

\section{Der Weg zur Welternährungssicherheit}

\subsection{Modernisierung und technischer Fortschritt}

Viele Limiten, die auf die landwirtschaftliche Produktivitätssteigerung und auf die Umsetzung wissenschaftlicher Erkenntnisse und technischer Innovationen hindernd wirken, lassen sich gemäss FAO aus Punkt 3.3. ableiten. Sie lassen sich soziokulturell begründen, sind bedingt durch strukturell bedingte institutionelle, politische und administrative Widrigkeiten und werden durch ökonomische Engpässe verstärkt. Die Überwindung solch struktureller Hindernisse würde das verfügbare Agrarpotential freigeben, den soziokulturellen Wandel begünstigen und schnelle Veränderungen in den technologischen Produktionsniveaus ermöglichen. Damit würden traditionelle Produktionsverhältnisse dem technologischen Wandel und der Einführung modernerer Produktionsformen Platz machen. Die FAO visiert dabei nicht eine hochtechnologisierte Landwirtschaft an. Diese birgt zu hohe ökologische Risiken in sich. Dem angepassten, mittleren Technologieniveau hingegen kommt zur Steigerung der Produktivität in den LIFDCs grösste Bedeutung zu. 


\subsection{Spezialprogramm für einkommensschwache Nahrungsdefizitländer (LIFDCs)}

Angesichts der 800 Millionen hungernden und unterernährten Menschen sieht die FAO in der Erhöhung der Ernährungssicherheit in den ärmsten Ländern ihr Hauptanliegen. Basierend auf dem dargestellten Analyseinstrument wurde in der Studie "Horizon 2010" das noch unausgeschöpfte landwirtschaftliche Potential der Entwicklungsländer ermittelt. Eine der Schlussfolgerungen der Studie: Gemäss FAO genügt das noch vorhandene landwirtschaftliche Potential für die Mehrheit der 88 LIFDCs, um ihren Bedarf an Nahrungsmitteln bis zum Jahr 2010 mehrheitlich selber decken zu können. Das Ziel der Ernährungssicherheit könnte somit erreicht werden. Dabei gilt allerdings, dass hierfür die heutigen agrartechnologischen Erfahrungen möglichst vollumfänglich für eine nachhaltige landwirtschaftliche Entwicklung ausgewertet werden müsste. Dies ist wiederum nur möglich, wenn die in Kapitel 3 beschriebenen limitierenden strukturellen Faktoren und Engpässe überwunden werden können.

In einer Sondersitzung des Rates vom November 1994 erhielt der neue Generaldirektor J. Diouf im Prinzip grünes Licht für die Lancierung eines "Spezialprogrammes für Nahrungsmittelproduktion zur Stärkung der Ernährungssicherheit in einkommensschwachen Nahrungsdefizitländern“. Mit limitierten finanziellen Mitteln sollte in ausgewählten LIFDCs ein neuer Anlauf zur partizipativen Planung und Umsetzung von Programmen, beruhend auf einem Modernisierungsansatz durch technologische Innovationen, genommen werden. Durch die Überwindung struktureller Hindernisse, durch die Mobilisierung von finanziellen Ressourcen und die Einbindung aller Partner (staatliche Stellen, Hilfsorganisationen, privater Sektor, Bauern) in Planung und Durchführung von Programmen sollte damit eine Trendwende in der Nahrungsmittelproduktion eingeleitet werden.

Die städtische Bevölkerung der LIFDC's wird sich gemäss Trendanalyse bis zum Jahr 2025 von einem Viertel auf die Hälfte des Bevölkerungsanteils vergrössern. Die zunehmende urbane Nachfrage muss über einen landwirtschaftlichen Überschuss gesättigt werden. Um das städtische Hungerproblem lösen zu können, müssen gemäss Spezialprogramm Agrarregionen in urbaner Nähe, sowie Marktstrukturen für moderne landwirtschaftliche Inputs und für die Vermarktung der Agrarprodukte gefördert werden.

Die Aufmerksamkeit der FAO gilt in erster Linie jenen agrar-ökologischen Regionen der LIFDC's, die mit einem hohen, noch wenig genutzten Ertragspotential ausgestattet sind (sogenannte high potential areas: HPAs). Auf Grund hoher Bedürftigkeit fällt der geographische Hauptakzent auf afrikanische Länder südlich der Sahara, bei denen die Prognosen bezüglich Bevölkerungs- und landwirtschaftlichem Wachstum Anlass zu besonderer Beunruhigung geben (siehe Tabelle). Mit erneuertem produktionstechnischem Effort gilt es, dem rasanten Bevölkerungswachstum zu begegnen. Beispiele sollen demonstrieren, dass Entwicklungshindernisse durch partizipative 


\section{Spezialprogramm}

Die FAO-Strategie des Spezialprogrammes basiert auf dem Ziel einer möglichst schnellen Erhöhung der Nahrungsmittelproduktion durch:

- Konzentration knapper Mittel auf ertragsstarke Regionen

- Einführung verbesserter angepasster Technologien unter bestmöglichem Einbezug der Zielgruppen bei der Wahl technologischer Innovationen

- raschen Technologiewandel mittels Demonstrationseffekten

- den Einsatz von haushälterischen Kleinbewässerungsmethoden inklusive fussbetriebener Pedalbewässerung

- die Partizipation aller Partner (staatliche Stellen, Hilfsorganisationen, privater Sektor, Bauern, NGOs)

Eine Pilotphase dient der Wahl der geeignetsten Technologie sowie der Identifikation limitierender Faktoren und Engpässe, welche der breiten und nachhaltigen Adaptation bewährter Technologien im Wege stehen.

In der Phase der Expansion rücken Reform- und Strukturverbesserungsmassnahmen zur Überwindung begrenzender Faktoren in den Vordergrund.

Weitere Elemente in der Expansionsphase sollen Investitionsvorhaben bilden wie:

Bewässerung (Neuanlage oder Wiederherstellung von Anlagen), ländliche Infrastruktur (Transport, Lagerung, Verarbeitung),

Produktion von und Belieferung mit landwirtschafltichen Betriebsmitteln

Schaffung institutioneller und organisatorischer Rahmenbedingungen wie Genossenschaftswesen, Kreditinstitutionen, Verarbeitungs, Lagerungs- und Vermarktungsstrukturen, Instandhaltungsdienste für Maschinen und Ausrüstungen, u.a.m.

Obwohl letztlich alle LIFDC's am Programm beteiligt werden sollen, sind aus administrativen und finanziellen Gründen vorerst nur 14 Länder in das Spezialprogramm aufgenommen worden. Die Leitung soll von den jeweiligen nationalen oder lokalen Behörden wahrgenommen werden, wobei das Landwirtschaftsministerium die Gesamtverantwortung übernehmen soll. (Afrika: Burkina Faso, Eritrea, Äthiopien, Guinea, Kenia, Mauretanien, Niger, Sambia, Senegal, Tansania; Lateinamerika: Bolivien; Asien: China, Nepal, Papua-Neuguinea)

Bei der Vorbereitung und Durchführung des Programmes will die FAO mit einer grossen Zahl externer Partner zusammenwirken. Die Gesamtleitung des Spezialprogrammes obliegt dem Generaldirektor, der hiefür über eine spezielle Leitungs- und Koordinationseinheit verfügt. 
Strategien zu überwinden sind, und dass damit die Erträge in noch wenig genutzten Regionen mit hohem Potential wesentlich gesteigert werden können. Den technologischen Impuls soll eine die Prinzipien der Nachhaltigkeit befolgende "Neue Grünen Revolution" vermitteln.

\subsection{Eine "Nachhaltige Neue Grüne Revolution"}

Der Grünen Revolution schenkte die FAO bereits in der Vergangenheit grosse Aufmerksamkeit. In Asien und Lateinamerika gelang es, damit spektakuläre Produktionssteigerungen zu erzielen. Schon im frühen Stadium machten allerdings namhafte Experten nicht zu Unrecht auf die ökologischen Gefahren einer allzu intensiven Bodennutzung aufmerksam. Daher wurde der Generaldirektor von den FAO-Gremien zur Vorsicht hinsichtlich des Einsatzes klassischer Strategien der Grünen Revolution ermahnt, deren Resultate oft im Widerspruch zu den Prinzipien der Nachhaltigkeit stehen. Um Fehler der Vergangenheit beim geplanten Technologietransfer nach Afrika zu verhindern, arbeitet die FAO, zusammen mit anderen Forschungsinstituten, am Instrument einer "Neuen Grünen Revolution“. Dieses soll im Rahmen des obengenannten Spezialprogrammes derart zum Einsatz kommen, dass bisherige Fehler ökologischer und sozial-ökonomischer Art vermieden werden können. Die Intensivierung der Landwirtschaft soll, in Übereinstimmung mit der UNCED Agenda 21, Kriterien der ökonomischen, ökologischen und sozialen Nachhaltigkeit standhalten können. Es herrscht aber immer noch Unklarheit darüber, wie dies zu geschehen hat. Der in letzter Zeit zurückhaltendere Gebrauch der Terminologie "Grüne Revolution" seitens der FAO weist allerdings darauf hin, dass die Idee einer Intensivierung der Landwirtschaft gerade auch im afrikanischen Raum realistischeren Überlegungen zur Machbarkeit gewichen ist.

Gerade in Afrika aber steht die Organisation unter einem besonderen Erfolgsdruck. Um in absehbarer Zeit dem Bevölkerungszuwachs von der Produktionsseite her zuvorzukommen, ist es daher durchaus gerechtfertigt, kurzfristig einen landwirtschaftlichen Produktionsschub anzuvisieren. Allerdings ist ein durchschlagen Erfolg nur bei Überwindung institutioneller, ökonomischer und sozio-kultureller Engpässe möglich. Die diesbezüglichen Erfolgschancen werden von Afrikakennern allerdings zurückhaltend beurteilt.

\section{Beurteilung und Vorschläge aus Sicht der Schweiz}

\subsection{Vorbemerkung}

Um der FAO mit einer Beurteilung des Sonderprogrammes gerecht zu werden, muss folgende Bemerkung vorausgenommen werden:

Oberstes Bestreben der FAO ist es, in absehbarer Zeit die Ernährungssicherheit in LIFDCs wesentlich zu verbessern, um damit das Ziel einer schnellen Reduktion von Hunger, Unter- und Fehlernährung zu erreichen. Die 
vorgelegte Strategie zur Überwindung von Engpässen basiert zum vornherein auf dem Handicap äusserster Limitierung finanzieller Ressourcen. Die vom FAO-Rat bewilligten Mittel für das Sonderprogramm beruhen auf internen Sparmassnahmen. Es ist fraglich, wie damit ein Spezialprogramm zur Bekämpfung des Hungers in vorerst 14 Ländern, selbst als vorbereitende Planungsphase, alimentiert werden kann. Eine umfassende, längerfristig konsistent angelegte Strategie zur nachhaltigen Verbesserung der Ernährungssicherheit scheint damit zum vornherein unmöglich zu sein. Eine solche aber benötigt eine Organisation, die über das Jahr 2010 hinaus die Ernährungssicherheit nachhaltig verbessern soll.

Jegliche kritische Argumentation könnte also mit der Begründung knapper Ressourcenverfügbarkeit von der Hand gewiesen werden. Im folgenden geht es dennoch darum, konstruktive realistische Anregungen zu einer längerfristig angelegten Strategie im Dienste der nachhaltigen Ernährungssicherheit darzulegen, wie sie die Schweiz in den FAO-Gremien zum Ausdruck bringt.

\subsection{Grundsätzliche Beurteilung}

Die FAO setzt in inrem Sonderprogramm auf noch wenig genutzte Hochertragsregionen. Zugleich errechnet sie, dass im Jahre 2010 die in den Entwicklungsländern noch vorhandenen kultivierbaren Landreserven von 90 Millionen Hektaren erschöpft sein werden. Im selben Zeitraum wird, gemäss geschätzten Verlusten von jährlich 5 bis 7 Millionen Hektaren, etwa gleich viel produktives Land verloren gehen. Die Frage liegt daher auf der Hand: Wie ist es um die Zukunft nach dem Jahre 2010 bestellt? Müssten nicht bereits heute Bodenverbesserungsmassnahmen Kernstück einer längerfristigen Strategie sein? Müsste nicht auch Land in den regenabhängigen, semi-ariden Tropen gemäss neuer Erkenntnisse über nachhaltige Bewirtschaftung von Böden mit geringer oder geschwundener Ertragskraft in ein Programm der Ernährungssicherheit einbezogen werden? Zur Erreichung kurzfristiger Erfolge läuft die FAO Gefahr, ihre eigenen diesbezüglich laufenden Programme hintanzustellen.

Wegen Knappheit an finanziellen Ressourcen steht also die Intensivierung der Landwirtschaft auf neuem Hochertragsland oder bereits erschlossenen Flächen mit hohem Ertragspotential im Zentrum der Strategie. Mit knappen Mitteln kurzfristig die höchsten Grenzerträge erzielen zu wollen, führt unweigerlich zum Einsatz gemäss Spezialprogramm. Sogenannte "fragile areas" wie Bergregionen, dürregeplagte Regionen, vormals bewaldete sowie schlecht unterhaltene, übernutzte Bewässerungszonen werden bei knappen finanziellen Ressourcen von Spezialinvestitionen ausgenommen. Obwohl sie sich kurzfristig für eine Steigerung der landwirtschaftlichen Produktion als nicht geeignet erweisen, anerkennt die FAO, dass angepasste Konservierungs- und Rehabilitationsmethoden existieren, die viele dieser Regionen in einen nachhaltig nutzbaren Zustand zurückführen könnten. Bodenverbesserungs- und Erosionsschutzmassnahmen sind aber schwierig finanzierbar. Unter den heutigen Vorzeichen des Rückgangs internationaler 
Entwicklungshilfe droht daher der Planet Erde einem Zustand entgegenzutreiben, der das genannte Dilemma der Menschheit am Übergang vom 20. zum 21. Jahrhundert noch verschärft.

\subsection{Migration Land-Stadt: der Fluchtweg Hungernder}

Wenn die Bekämpfung des Hungers oberste Zielsetzung der FAO ist, dann muss sie an den Wurzeln, bei den Ursachen ansetzen. Das vorgelegte Spezialprogramm ist explizit auf die Versorgung einer stark zunehmenden städtischen Bevölkerung ausgerichtet. Die Ursachen des Trends zum Illusionszentrum Stadt sind zum grossen Teil bekannt. Sie liegen in der falschen Prioritätensetzung mancher Regierungen hinsichtlich Privilegierung von Investitionen in urbanen Zentren und einer oft falsch geplanten, prestigeorientierten Industrialisierungspolitik. Armut und Hunger sind aber in erster Linie in ländlichen Regionen weit verbreitet. Wenn sich diese Armut im ländlichen Raum wegen unterlassener Entwicklungsinvestitionen weiter perpetuiert, kann auch die Migration in die urbanen Zentren, die nichts anderes als der Ausdruck verzweifelter Suche nach einer verbesserten existentiellen Sicherheit darstellt, nicht aufgehalten werden. Nur eine Verbesserung der Lebensbedingungen im ländlichen Raum kann in den LIFDCs eine Trendwende bewirken. Der von der FAO vorschnell als Faktum akzeptierte Trend der Land-Stadt Migration wird durch die dargelegte Strategie noch verstärkt.

Nicht nur lebt die Mehrheit der 800 Millionen Hungernden in ländlichen Räumen, sondern zugleich in jenen Landwirtschaftszonen, die in den Land Surveys der FAO-Studie 2010 als wenig produktive Böden klassifiziert sind. Von den 900 Millionen Menschen in den regenabhängigen semi-ariden Tropen, die als "Heimstätten der Armen und Hungernden" gelten, leben $\mathbf{3 0 0}$ Milionen Menschen in absoluter Armut. Insbesondere in Afrika kann die landwirtschaftliche Produktion dieser Zonen nicht mit dem Bevölkerungswachstum Schritt halten. Auf Grund der sehr beschränkten Bewässerungsmöglichkeiten in den semi-ariden Tropen bietet das Spezialprogramm der FAO diesen Einwohnern kaum die Chance eines verbesserten Ernährungsstatus. Vermehrte Investitionen in die Entwicklung der regenabhängigen semi-ariden Tropen und in gefährdete Wassereinzugsgebiete sind aber klar sich stellende Herausforderungen (siehe Beispiel Indien).

Will die FAO also der Armut und dem Hunger die Stirne bieten, dann muss sie die genannten Elemente in ihre Strategie zur Verbesserung der Ernährungssicherheit aufnehmen. Selbst wenn vorerst keine grossen vermarktbaren Überschüsse aus den semi-ariden Regionen zu erwarten sind, müssen die Bäuerinnen und Bauern dieser Hungerzonen das neueste, für sie relevante landwirtschaftliche Wissen vermittelt erhalten. Subsistenzbauern können mit verbessertem Know-how ihre Existenzgrundlage entscheidend verbessern, ihre chronische Unterernährung hinter sich lassen und allmählich sogar vermarktbare Überschüsse erzeugen. 


\section{Neue partizipative Methoden in semi-ariden Gebieten Indiens}

Am Ende einer über 30-jährigen Phase erfolgreicher landwirtschaftlicher Steigerung der Produktion mittels Grüner Revolution ist Indien an jenem Punkt angelangt, an dem die Grenzerträge in den klassischen Bewässerungsgebieten abzunehmen beginnen. Versalzung, Auslaugung und Verlust ehemals fruchtbaren Ackerlandes durch Übernutzung haben sichtbare Ausmasse angenommen.

In dieser Situation setzt Indien relativ spät auf die Entwicklung lange Zeit vernachlässigter semi-arider Regionen, in denen die Ärmsten der Armen leben, welche kaum oder gar nicht von den Vorteilen der GR profitiert haben. Diese regenabhängigen semi-ariden Tropen bedecken über $50 \%$ Indiens. Sie sind die Heimstätte der Ärmsten, die wenig oder gar keine Kaufkraft besitzen. Das sogenannte "Watershed Development Programme" zur Verbesserung der Ökologie in Wassereinzugsgebieten der dürregefährdeten Regionen hat zum Ziel, die Produktivität dieser Regionen im Hochland des Dekans mittels ökologisch, ökonomisch und sozial nachhaltiger Entwicklungsinvestitionen zu steigern.

In Zusammenarbeit mit Forschungsinstituten wie ICRISAT und unter Einbezug von Nichtregierungsorganisationen ist es der Regierung in Maharashtra und Karnataka bereits gelungen, in Regionen mit sehr niedriger Ertragskraft Programme zur partizipativen Entwicklung von Wassereinzugsgebieten mit folgenden Komponenten zu lancieren:

- sog. Wasserernte-Strukturen zur Revitalisierung des Grundwassers und zur Regenerierung der Vegetation.

- Bodenverbesserungsmassnahmen durch Gründüngung und durch den Einsatz von Biogas-Anlagen

- integrierte Aufforstungsmassnahmen zur Eindämmung der Erosion, zum Schutz des Wassers und als Futterbasis

- Einsatz trockenresistenter Saatsorten (insbes. Hirsesorten) in Zusammenarbeit mit ICRISAT. (International Crops Research Institute for the Semi-Arid Tropics, Hyderabad)

- Breitabgestützter demokratischer Einbezug der Bevölkerung bei Planung, Wiederaufbau und nachhaltiger Nutzung von Wasser, Land und Forst, sowie zum Aufbau und zum Unterhalt neuer Infrastrukturen etc.

Solche Programme erfordern hohe Investitionen. Die Dorfgemeinschaften und Bauernfamilien tragen durch Fronarbeit zum Wiederaufbau bei, erhalten aber auch für erbrachte Normleistungen monetäre Abfindungen und Nutzungsrechte an Weiden und Wäldern. Die Zahlungen der indischen Regierung werden von der deutschen Bundesregierung, der Schweiz und anderen Ländern mitgetragen. 
Eine weitere nennenswerte Entwicklung ist jene in von Bewässerungssystemen nicht erschlossenen Regionen, die aber gute Grundwasserverhältnisse oder Flussläufe aufweisen. Hier haben NGOs, worunter auch schweizerische, damit begonnen, abseits grosser Regierungsprogramme und noch weitestgehend unbemerkt von diesen, Kleinbewässerungsmethoden einzuführen, bei denen mittels fussbetriebener Bewässerungspumpen ein sparsamer, nachhaltiger Umgang mit Wasser aus Flüssen, Seen, Kanälen und Grundwasser es erlaubt, den Bewässerungsperimeter auch in Regionen mit geringerer Ertragskraft wesentlich zu erweitern.

Beide Massnahmen werden die Ernteerträge Indiens weiter erhöhen und die Ernährungssicherheit in marginalen Regionen wesentlich steigern. Ähnliche Beispiele sind auch in andern Ländern Asiens und in Afrika im Aufbau begriffen. Ein Süd-Süd Erfahrungsaustausch, von der FAO mitgetragen, könnte den Prozess wesentlich beschleunigen.

\subsection{Hunger, auch ein Problem der Kaufkraft}

Die Kausalitätskette von Armut, mangelnder Kaufkraft, Hunger, sinkender Arbeitsproduktivität, mangelnder ruraler Ersparnisse, Unterinvestitionen in Bodenfruchtbarkeit und in verbesserte Anbaumethoden muss an mehreren Stellen zugleich aufgebrochen werden. Die zentrale Frage von Hunger mangels Kaufkraft, im Spezialprogramm nur beiläufig erwähnt, wird im Jahre 2010 von noch drängenderer Aktualität sein. Indem die FAO die Hungerproblematik kurzfristig produktionsorientiert zu lösen versucht, läuft sie Gefahr, sie aus ökologischen und ökonomischen Gründen längerfristig erst recht zu verstärken.

Wenn heute 800 Millionen Menschen unterernährt sind, so ist das nebst der Folge zu knapper Agrarproduktion auch der Ausdruck mangelnder Kaufkraft der Armen. In zweiter Hinsicht ist es auch ein Problem mangelnder Vermittlung von Produktionsanstössen und Investitionen in Know-how bei jenen Subsistenzbauern, die über nur geringe Mittel und eigene Möglichkeiten verfügen, um ihre Produktions- und Selbstversorgungsbasis zu verbessern. Die Frage, ob denn dieArmen und Subsistenzbauern - abseits von Agrarmärkten lebend und bar der minimalsten Kaufkraft - mit einer technologischen Modernisierungsstrategie unvermittelt in den Marktprozess einer landwirtschaftlichen Produktivitätsteigerung einbezogen werden können, muss allerdings mit "Nein" beantwortet werden. Das Nein liegt zu einem ansehnlichen Teil im zeitlichen Erfolgsdruck, unter den sich die FAO selber begibt, begründet. Nichts aber führt für den Aufbau bisher vernachlässigter Regionen an einem längerfristig ökologischen, ökonomischen und sozial 
partizipativen Entwicklungsprozess vorbei. Für die 40 Prozent der Ärmsten in Indien zum Beispiel, einem Land, das Nahrungsmittel exportiert, beginnt dieser partizipative Prozess in jüngster Zeit reiche Früchte zu tragen. Doch solche demokratischen Prozesse erfordern Zeit.

\section{Schlussfolgerungen}

Die FAO wird zur langfristigen Ernährungssicherung auch den wenig ertragreichen Böden grösste Aufmerksamkeit schenken müssen. Eine langfristig ausgerichtete Strategie nachhaltiger Ernährungssicherheit nimmt sich auch jener 2 Milliarden Hektaren Bodens an, die in den letzten 50 Jahren degradiert wurden. Hiervon könnten ca. 80 Prozent leicht- und mitteldegradiertes Land durch Zusatzinvestitionen auf Bauernbetrieben rehabilitiert werden, während der Rest stark degradierten Landes mit Massnahmen im Sinne des Watershed Development Programmes zurückgewonnen werden könnten.

Eine Mehrheit der armen Bevölkerung der Entwicklungsländer lebt in diesen semi-ariden und gebirgigen Gebieten. Keine Strategie der Hunger- und Armutsbekämpfung kommt darum herum, der Bevölkerung dieser Regionen hohe Priorität einzuräumen. Dies selbst dann, wenn vorerst nur ein Teil dieser Bauern in der Lage sein wird, urbane Zentren mit bedeutenden Marktüberschüssen zu versorgen. Wenn diese Bevölkerungsgruppen aber weiterhin verstärkt abwandern, tragen sie unweigerlich zur urbanen Nachfrage bei.

Diese Regionen daher einzubeziehen in eine Strategie zur Verbesserung der Ernährungssicherheit, muss Bestandteil werden eines FAO-Programmes ländlicher Entwicklung. Eine verstärkte Zusammenarbeit mit den diesbezüglich erfolgreichen CGIAR-Forschungszentren drängt sich auf. Ebenso ist ein Erfahrungsaustausch zwischen Entwicklungsländern auf dem Gebiet der Entwicklung und Regenerierung von Wassereinzugsgebieten (Watershed Development) von grosser Dringlichkeit. Die FAO verfügt über finanzielle Mittel zur Verbesserung der Süd-Süd Zusammenarbeit zwischen Entwicklungsländern.

Nur mit einer ausgewogenen Entwicklungsstrategie zwischen Regionen mit hohem und solchen mit niedrigem Ertragspotential wird eine nachhaltige Ernährungssicherheit in den LIFDCs erreicht werden können. Unterernährung und Landflucht müssen notgedrungen auch in den marginalen Regionen der Armut und des Hungers angegangen werden. Dies erfordert langfristige Investitionen im Kampf gegen Bodenübernutzung, Erosion und Verwüstung. Die zwei Entwicklungspole des technischen Fortschritts in Hochertragsregionen einerseits und der landwirtschaftlichen Innovation und Erneuerung natürlicher Ressourcen in weniger produktiven Regionen andererseits sind komplementär. Eine in diesem Sinne zweifach Grüne Revolution resultiert in einer dauerhaften Reduktion von Hunger und Armut. In einem partizipativ gestalteten Entwicklungsprozess führt sie hin zu einer ökonomisch, ökologisch und sozial nachhaltigen Ernährungssicherheit. 
FAO-Beiträge der Schweiz (in Mio US \$)

\begin{tabular}{|l|r|r|r|r|}
\hline & 1992 & 1993 & 1994 & 1995 \\
\hline Ordentliche Jahresbeiträge & 4.21 & 4.17 & 4.13 & 4.13 \\
Projektbeiträge & 6.8 & 6.8 & 6.2 & \\
\cline { 2 - 5 } Total & 11.01 & 10.97 & 10.33 & \\
\hline
\end{tabular}

